\title{
A Study on SSO Restraint and Coordination Control under Rapid Development of Clean Energy
}

\author{
Xiaoqing Yan and Xinting Huang
}

\begin{abstract}
The contrary distribution between Clean Energy Base and load center of China has pushed the rapid development of UHV transmission, and SSO (Subsynchronous Oscillation) caused by multiple HVDC (High Voltage Direct Current) power transmission system has been more and more concerned by experts and scholars. Subsynchronous damping controller (SSDC) has been one of the most common restraint measures. The coordination control of SSDC is investigated in this paper, in the background of the alternative-direct transmission power system of Guizhou province. Three conclusions are verified by theoretical analysis and numerical simulations. Firstly, the SSDC and subsynchronous excitation damping controller (SEDC) are decoupled and set separately in the SSO restrain. Secondly, the coordination control of SSDC in different converter stations is necessary. At the same time, the generator speed can be applied as input signal for an effective restrain with the rapid development of communication technology. The advices proposed in this research can be helpful to the rapid development of HVDC transmission system.
\end{abstract}

Index Terms-Subsynchronous oscillation, HVDC power transmission system, Subsynchronous damping controller, Subsynchronous excitation damping controller.

\section{INTRODUCTION}

With continuous construction of large-scale renewable energy bases and ultra-high voltage grids, the DC power transmission technology is more and more concerned. In the High Voltage Direct Current (HVDC) transmission system, subsynchronous oscillation (SSO) occurred in large generator unit has become one of the problems that could not be neglected since it causes severe impact on the stable operation of the system and the service life of the unit shaft. HVDC converter control system may cause SSO in together with the torsional vibration negative feedback of large unit shaft nearby. This problem is initially identified in the Square Butte DC power transmission project [1], and the CU and IPP projects in the United States and some of other HVDC power transmission projects all show the occurrence possibility of SSO.

The subsynchronous oscillation (SSO) caused by the rapid adjustment system of HVDC is commonly restrained with subsynchronous damping controller (SSDC) and a series of research findings have been obtained. Research [2], [3], primarily describes a relatively complete design concept and control mode, which paves a solid way for follow-up research.

Manuscript received October 21, 2015; revised March 9, 2016.

Xiaoqing Yan is with the Energy Research Institution of State Grid, Beijing, China (e-mail: yanxiaoqing@ sgeri.sgcc.com.cn).

Xinting Huang is with the China Power Finance Co., Ltd., Beijing, China (e-mail: dearhxt@139.com).
References [4]-[10] emphasizes several common methods for SSO analysis and restraint. Research on SSDC has also conducted in China. SSDC parameter design with genetic algorithm is proposed, then the adjust measure of SSDC with robust control theory based on $\mathrm{H}$ positive infinity is established [11], [12].

Based on the field operation of Guizhou power grid with much HVDCs, appropriate thermal generators as research object are selected with unit interaction factor method(UIF), then the equivalent network that can rationally reflect the relationship between SSO and HVDC system is established based on which, conducted a lot of simulation research on the coordination control of SSDC and SEDC (Subsynchronous Excitation Damping Controller) in multiple HVDC power transmission system and the coordination control of multiple SSDC. Therefore, some accomplished explorations of effective SSO restraint are proposed in the last chapter.

\section{RESEARCH BACKGROUND}

It has been confirmed and acknowledged that the internal correlation between SSO occurrence and the feature of HVDC control system is the commonest mechanism of generation. When electric negative damping exceeds mechanical damping of shaft, the negative feedback between unit rotation speed and electromagnetic torque would become even more intensified, which would not be covered herein.

Guizhou power grid has gradually, centered at 500kV loop network in central Guizhou, formed $500 \mathrm{kV}$ main network extending northward and eastward and $500 \mathrm{kV}$ "Five - $\mathrm{AC}$ and Two-DC Line" for power transmission from Guizhou To Guangdong, and China Southern Power Grid has also highly concerned SSO caused by multiple HVDC. Siemens has ruled out the possibility of SSO after a lot of research work on SSO occurrence caused by HVDC in Tian-Guang HVDC transmission project and, while for Gui-Guang II HVDC project, a large number of steam generator units are found nearby DC rectifier side and researches show that SSO would occur when DC system is designed with step-down under light-load (70\% of rated voltage and of active power) and provided with weak grid connection (or controlled islanding).

To reflect the engineering significance and practical value of the research, the author "screened" an equivalent system of Guizhou power grid and the modeling complexity with unit interaction factor method (UIF) and, on the basis of main AC loop network of Guizhou power grid, Gui- Guang I HVDC (Gao-zhao DC) and Gui-Guang II HVDC (Xing'-An DC), discovered relatively high degree of coupling between Pannan Power Plant, Anshun Power Plant and DC power transmission system. Therefore the complete generator model is necessary, while the simplified model may be adopted for 
other power plant units. Fig. 1 presents the equivalent simplification system of Guizhou power grid under main operation mode in wet season on which simulation study is made herein.

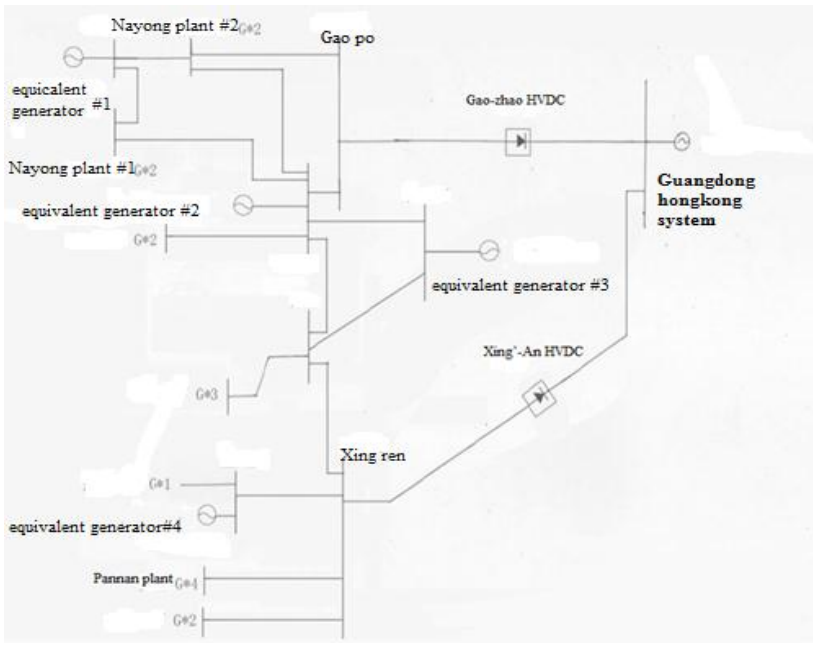

Fig. 1. Equivalent power system of Guizhou province.

\section{SSDC COORDINATION CONTROL}

\section{A. Selection of Control Parameters}

Generally SSO is currently restrained with SSDC in HVDC system in China, mainly including the synchronism link, smoothing link and phase correction link. Commonly SSDC would firstly take sample from AC voltage signals at convertor station busbar side, and in further get positive equivalent electrical damping value by regulating the current in DC system, and thus restricting subsynchronous resonance.

It is worth noting that SSDC would serve as secondary control equipment for practical engineering purpose. After installation and commissioning, usually SSDC control parameters regarding each link would not change along with system condition, and maintain constant during use. Each parameter therefore should have relatively strong adaption and robustness. Since the inhibition effect caused by SEDC and SSDC is completely decoupled (detailed in section B), firstly the optimal solution of control parameters during phase shift link regarding SEDC and SSDC controller is confirmed with genetic algorithm. The target function may be designed as the eigenvalues of control system far away the left side of complex plane as much as possible; as to the coordination control of multiple SSDC (detailed in section C), it is required to compare and select the optimal solution through intensive time-domain simulation analysis following respective parameter setting.

\section{B. Coordination Control of SSDC and SEDC}

Subsynchronous excitation damping controller (SEDC), currently one of the most common approach to restrain SSO, deems steam turbine's high-pressure cylinder rotation speed signal as feedback signal, gets mode functions of SSO with band-pass filter, and controls SEDC output signal by providing appropriate phase correction signal. Supplementary electromagnetic torque would increase the system's positive electric damping as long as parameter setting in SEDC control link is proper. SEDC meanwhile focus on the control at excitation system side, therefore the significantly increased investment and prominent economic strength is one of the reason for its widespread application.

In terms of physical process, both SEDC and SSDC are to restrict SSO by generating supplementary electromagnetic torque in respective control loop and their difference lies in the fact that the former's output signal is superimposed as supplementary exciting voltage onto exciting winding to provide supplementary electromagnetic torque, while the later provides supplementary electromagnetic torque through the fixed-current control loop at convertor station rectifier side. The vector sum of two supplementary electromagnetic torques on complex plane decides the system's electric damping and therefore, both is decoupled when superposition relation is satisfied by electromagnetic torque generated by different supplementary loops.

It is discovered in this paper based on intensive research, the positive electric damping provided by SEDC and SSDC is decoupled, satisfying linear superposition relation, namely to select the optimal parameter respectively during practical engineering, no need to conduct coordination control for SEDC and SSDC parameter setting and adjustment respectively at generator side and DC system side.

As to SSO caused by HVDC rapid control system, large-size generator unit is strongly coupled with convertor station under islanding mode, easily causing SSO divergence. Since Pannan Power Plant disconnects from Guizhou power grid electrically and operates under islanding mode by directly delivering power to Guangdong power grid via Xing'An DC (namely Pannan islanding mode, the same as below), the optimal SEDC and SSDC parameter is respectively set, and the electric damping factors under four operation modes are calculated, namely the mode without SEDC and SSDC, only SEDC configured, only SSDC configured and both SEDC and SSDC configured. As shown by in Fig. 2, the electric damping factor when only SEDC configured (namely the green curve) and only SSDC configured (the red curve) after linear superposition are approximately consistent with that when both SEDC and SSDC configured (light blue curve), indicating that the electric damping added by SEDC and SSDC device can be linearly superposed.

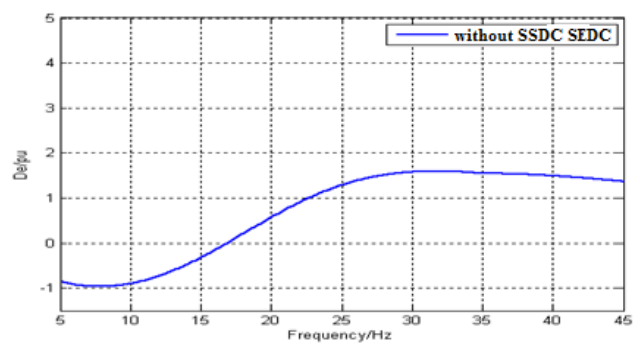

(a)

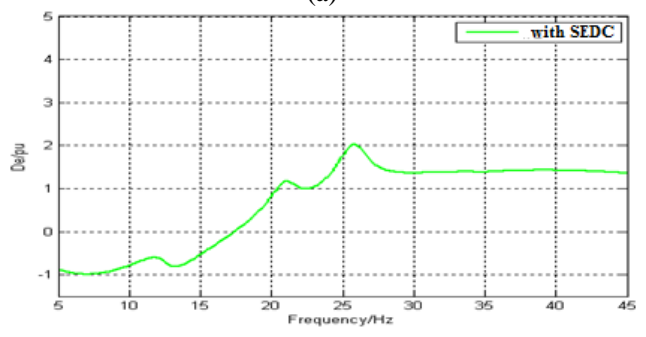

(b) 


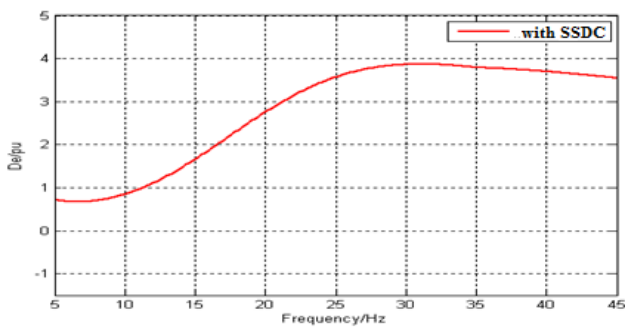

(c)

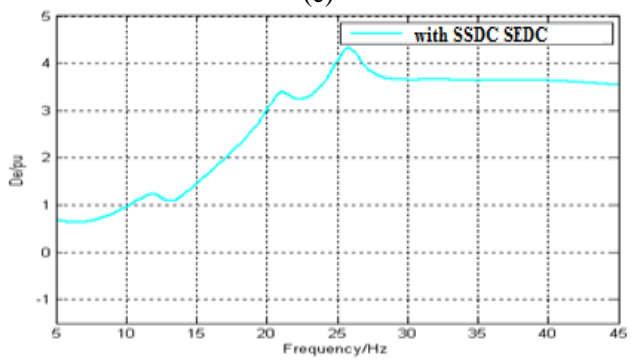

(d)

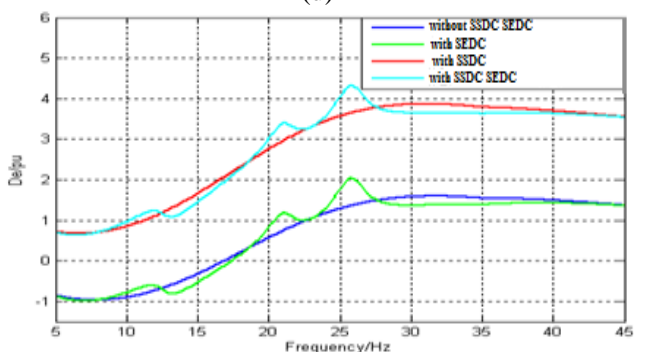

(e)

Fig. 2. The characteristic of electrical damping with SSDC/SEDC control.

\section{Multiple SSDC Coordination Control}

Gradual divergent SSO would be caused by small disturbance and also by transient torque amplification after large disturbance, the fundamental cause of which is that the phase difference between electromagnetic torque and generator shaft rotation speed is larger than 90 degree, causing negative damping of SSO. The core of configuring SSDC device therefore lies in the setting of phase compensation link, while in a power transmission system with multiple HVDCs, SSDC installed at line-side DC convertor station would respectively provides an additional electromagnetic torque for generator unit. The combine electric damping characteristics is decided by initial and Supplementary electromagnetic torque.

Obviously, the setting of single SSDC would no longer be an isolated issue, only coordination control can most effectively restrain SSO. The common method in practical engineering application is that, as mentioned above, its parameters would not change along with system state, operation mode and fault types, and remain changeless during operation, therefore it is required to conduct a large number of simulation experiments to select optimal parameter for SSDC coordination control, so as to restrain SSO under different working conditions.

As shown in Fig. 1, a simulation model is presented for Guizhou power grid operation in wet season, in which SSDC parameters are set with generic algorithm, optimal parameter is selected based on a large number of PSCAD time-domain simulation under different fault conditions, and the model specifically indicates the significance of SSDC coordination control in multiple HVDC power transmission system. The generator unit in Pannan power plant is selected as research object and, under the operation in wet season, the line from Pannan to Xingren convertor station is set to be subject to three-phase failure, and the most representative simulation result is selected.

Fig. 3 presents the SSO caused by transient torque amplification without SSDC and of which, Fig. 3(a) provides the rotation speed difference attenuation curve of the generator unit in Pannan power plant under three SSO modes, namely attenuation rate under mode 1,2 and 3 respectively being $-0.0741,-0.1462$ and -0.1823 , wholly slow convergence, causing sustainable impact on unit shaft system with relatively large amplitude value.

Fig. 3(b) shows the unit rotation speed difference while SSDC at Xing'an DC and Gaozhao DC is not subject to coordinated setting but provided with separately set control parameters. Fig. 3(c) provides unit rotation speed difference under each mode while two SSDCs are provided with coordinated setting parameters. It can be easily found after comparison with Fig. 3(b) that SSO under each mode has been restrained to a greater extent, and also verified the significance of SSDC coordination control in multiple HVDC power transmission system.
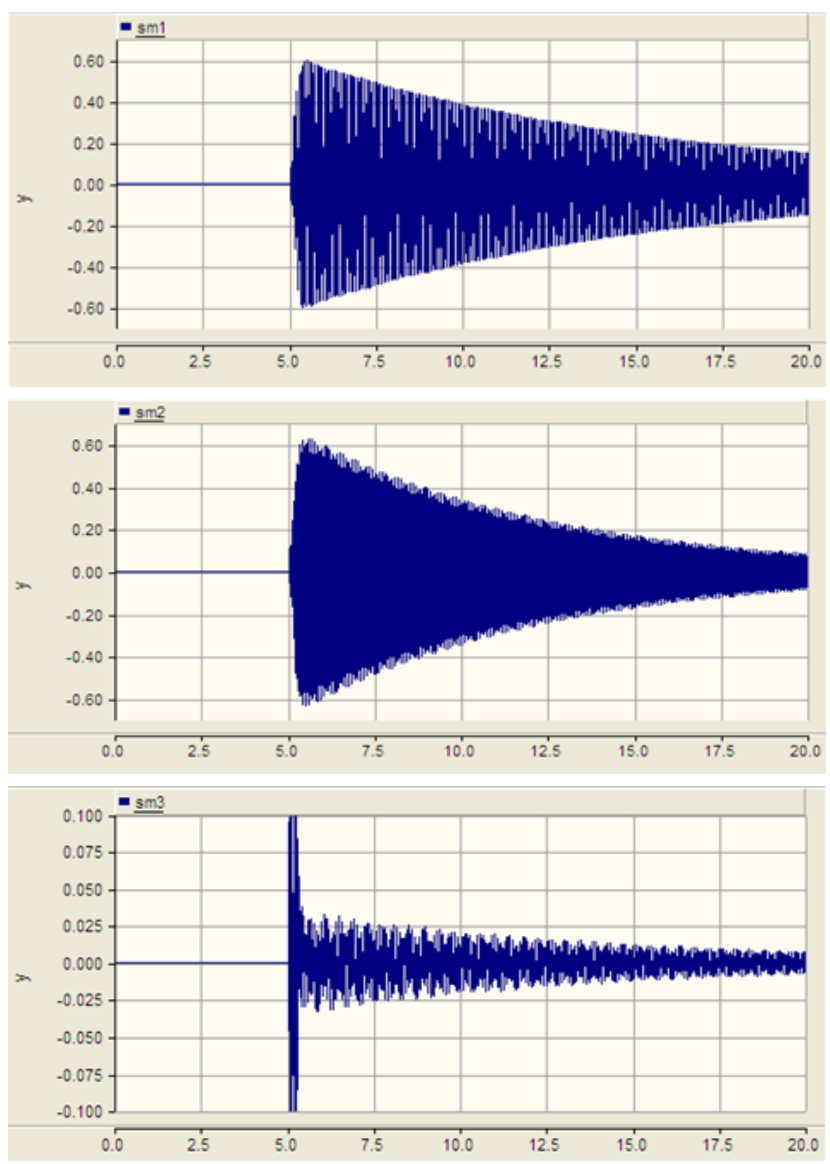

(a)

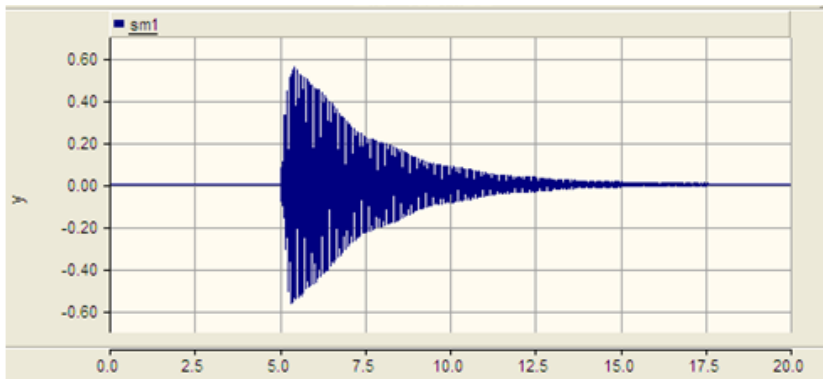



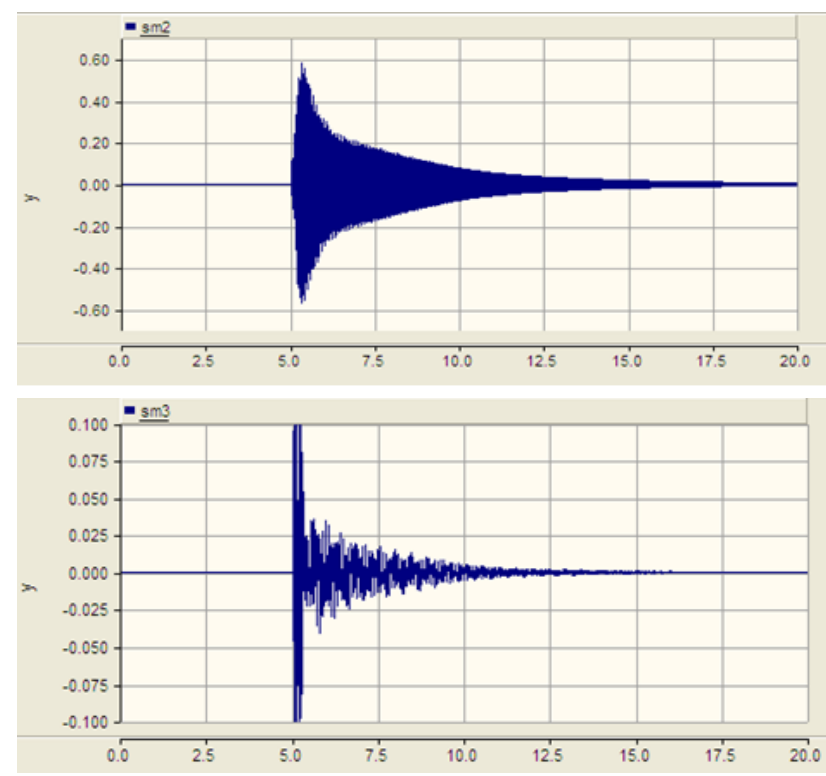

(b)
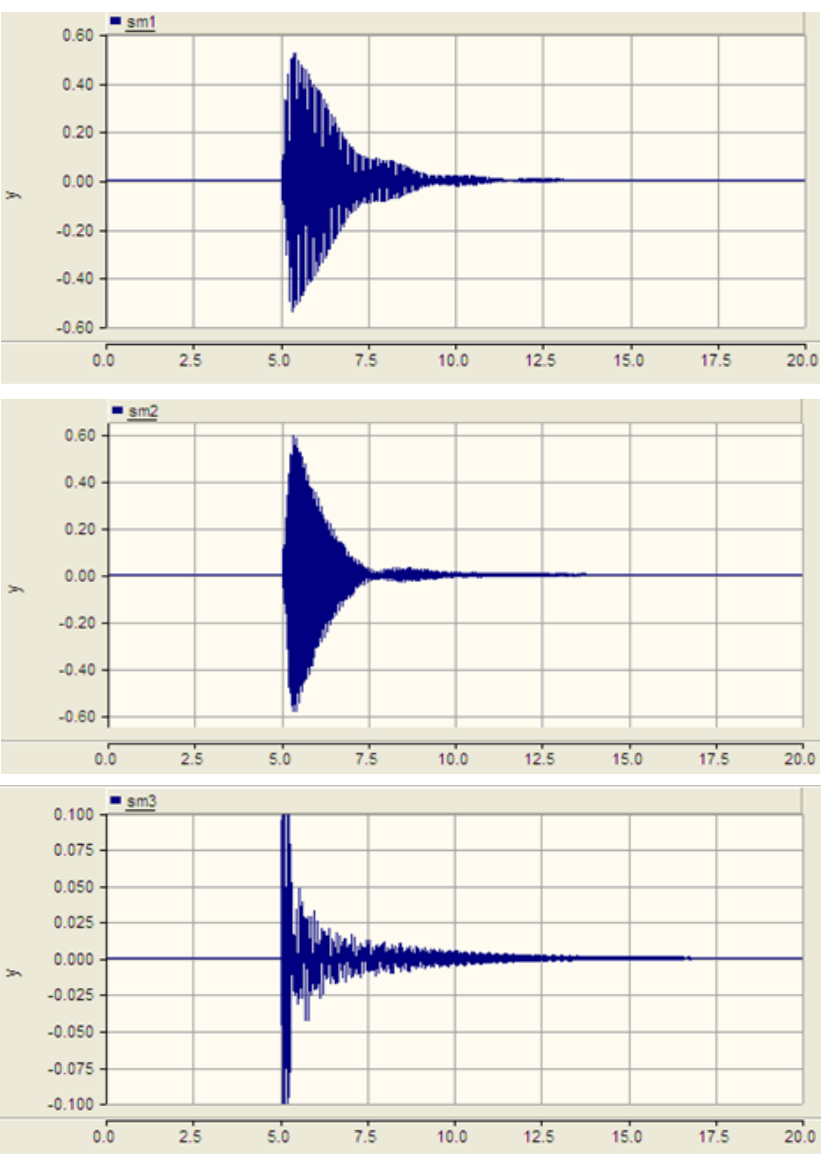

(c)

Fig. 3. The speed difference characteristic of unit shaft system with/without SSDC control.

\section{SENSITIVITY ANALYSIS}

The selection of SSDC input signal is an important influencing factor and generally, the generator unit's rotation speed signal or convertor station busbar AC voltage signal would be selected. Considering the far distance from high voltage DC convertor from power generation plant, the problems regarding signal transmission and reliability would occur. Currently AC voltage from convertor station generally serves as input signal in DC power transmission project.
SSO signal, however, would be submerged with noise when small disturbance is found in the system, the signal cannot be extracted by SSDC, the restraint on SSO generated by small disturbance would be limited. As to Pannan power plant unit, the synchronizing signal and noise signal in subsynchronous band (the non-synchronous frequency component with largest amplitude in subsynchronous band is defined as noise signal) is analyzed respectively for Guizhou power grid under normal operation mode and for Pannan under islanding mode. Obviously, SNR of $13 \mathrm{~Hz}$ subsynchronous frequency component under full-line mode is only $0.16 \%$, roughly submerged with noise signal, in particular the restraint on SSO caused by small disturbance is very limited. As to Pannan power plant under islanding mode, SNR of $29 \mathrm{~Hz}$ subsynchronous frequency component is up to $22.9 \%$, conductive to normal extraction of busbar voltage signal.

As communication technology becomes increasingly matured, the speed and reliability of signal transmission would be significantly improved, and taking generator unit rotation speed as SSDC input signal is more conductive to SSO restraint, particularly for SSO caused by small disturbance (see Fig. 4 and Fig. 5).

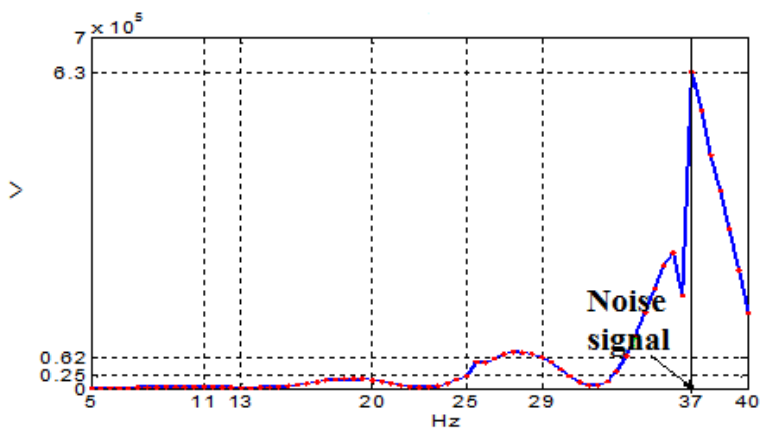

Fig. 4. The amplitude-frequency characteristic of bus voltage under normal operation state.

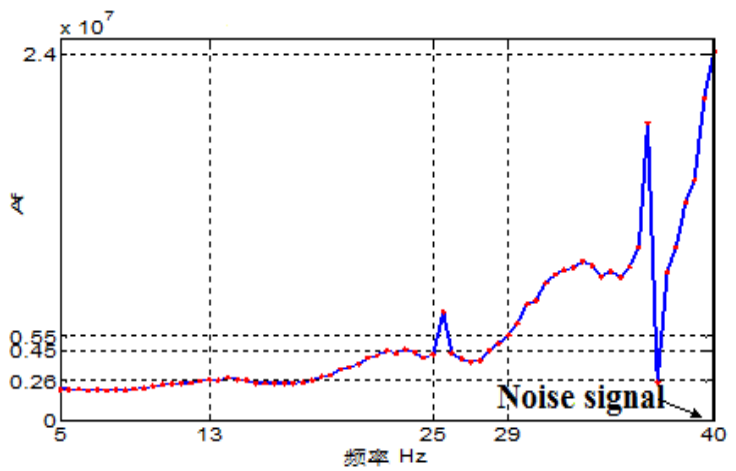

Fig. 5. The amplitude-frequency characteristic of bus voltage under island operation state.

TABLE I: ANALYSIS OF SIGNAL-NOISE RATIO

\begin{tabular}{llll}
\hline \hline Model frequency $(\mathrm{HZ})$ & \multicolumn{1}{c}{13} & \multicolumn{1}{c}{25} & \multicolumn{1}{c}{29} \\
\hline Full line mode & 0.0016 & 0.039 & 0.098 \\
Islanding mode & 0.108 & 0.1833 & 0.2291 \\
\hline \hline
\end{tabular}

\section{CONCLUSION}

SSO in HVDC power transmission system has been more and more concerned and, based on the practical engineering 
and with Guizhou power grid as simulation background, relevant study has been conducted on the coordination control of multiple SSDC in multiple HVDC power transmission system. The result demonstrates that:

1) Supplementary electric damping provided by SEDC and SSDC is decoupled, satisfying linear superposition relation, namely to select the optimal parameter respectively during practical engineering, no need to conduct coordination control for SEDC and SSDC parameter setting and adjustment respectively at generator side and DC system side.

2) In a multiple HVDC power transmission system, the setting of multiple SSDC installed at line-side DC convertor station would no longer be an isolated issue, only coordination control and parameter selection can most effectively restrain SSO.

3) As communication technology becomes increasingly matured, taking generator unit rotation speed as SSDC input signal is more conductive to SSO restraint.

Large number of simulation have verified the opinions proposed herein, and this paper is designed to play a certain role in promoting future construction and development of HVDC power transmission system in China.

\section{REFERENCES}

[1] M. P. Bahrman, E. V. Larsen, R. J. Piwko et al., "Experience with HVDC turbine generator torsional interaction at square Butte," IEEE Trans on Power Apparatus and Systems, vol. 99, no. 3, pp. 966-975, 1980.

[2] R. J. Piwko and E. V. Larsen, "HVDC system control for damping of subsynchronous oscillations," IEEE Trans on Power Apparatus and Systems, vol. 101, no. 7, pp. 2203-2211, 1982.

[3] Y. Y. Hsu and L. Wang, "Modal control of an HVDC system for the damping of subsynchronous oscillations," IEE Proceedings, vol. 136, no. 2, pp. 78-86, 1989.

[4] Z. Xu, R. Q. Luo, and R. J. Zhu, "Review on methods of analysis for subsynchronous oscillations of power systems," Power System Technology, vol. 23, no. 6, pp. 36-39, 1999.
[5] Y. Yang, R. Hu, C. Hong, and C. L. Ren, "The subsynchronous oscillation problem and the damping control strategy at the monopolar operation stage of Xingan HVDC transmission project," pp. 25-30, 2007.

[6] Y. Feng, "HVDC system control for damping of subsynchronous oscillation and its analysis software development," Shanghai Jiaotong University, 2008.

[7] D. Liu and L. Wang, "The solution of subsynchronous oscillation (SSO) in power plant," Sichuan Electric Power Technology, vol. 35, no. 2, pp. 78-84, 2012

[8] C. C. Zhou and Z. Xu, "Analysis of damping characteristic of the subsynchronous oscillation in AC/DC systems with series capacitor compensation," High Voltage Engineering, vol. 30, no. 2, pp. 1-3, 2004.

[9] J. Y. Fang, "Analysis of subsynchronous oscillation caused by HVDC and the damping controller design," North China Eclectic Power University, 2010.

[10] L. C. Li and C. Hong, "Analysis of the SSO problem caused by Guizhou-Guangdong II HVDC transmission system," Automation of Electric Power Systems, vol. 31, no. 7, pp. 90-93, 2007.

[11] Q. Y. Jiang, S. J. Cheng, and Y. J. Cao, "Design of HVDC supplementary subsynchronous damping controller genetic algorithms," Proceedings of the CSEE, vol. 22, no. 11, pp. 87-91, 2001.

[12] X. Yang, X. T. Wang, and C. Chen, "Design of supplemental subsynchronous oscillation damping control based on $\mathrm{H} \infty$ roust control theory of HVDC system," Power System Technology, vol. 30, no. 9 , pp. 57-61, 2006.

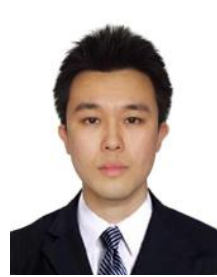

Xiaoqing Yan was born in 1986, Tianjin, China. He got his doctor degree from North China Electric Power University of China in 2013. His research interest is power system relay protection and control.

$\mathrm{He}$ is working in the State Grid Energy Research Institution of China. His research includes the power system planning, power balance analysis etc. Some of his research was published as journal paper and report.

Xinting Huang was born in Anhui Province, China. She received her MSc degree in finance from Warwick University in 2012. Currently, she is with China Power Finance Co., Ltd. Her main research interest is risk evaluation in energy and power department. 